\title{
Highly efficient cellular labeling of mesoporous nanoparticles in human mesenchymal stem cells: implication for stem cell tracking
}

\author{
Dong-Ming Huang, ${ }^{*}$ Yann Hung, ${ }^{\dagger}$ Bor-Sheng Ko, ${ }^{\ddagger}$ Szu-Chun Hsu, ${ }^{\ddagger}$ Wei-Hsuan Chen, ${ }^{*}$ \\ Chung-Liang Chien, ${ }^{\S}$ Chih-Pin Tsai, ${ }^{\dagger}$ Chieh-Ti Kuo,* Ju-Chiun Kang,* Chung-Shi Yang, \\ Chung-Yuan Mou, ${ }^{\dagger}$ and Yao-Chang Chen*, I, \\ *The Stem Cell Research Center, National Health Research Institutes, Miaoli, Taiwan, ${ }^{\dagger}$ The \\ Department of Chemistry, National Taiwan University, Taipei, Taiwan, ${ }^{\ddagger}$ The Department of Internal \\ Medicine, National Taiwan University Hospital, Taipei, \$ The Department of Anatomy and Cell \\ Biology, College of Medicine, National Taiwan University, Taipei, Taiwan, The Center for \\ Nanomedicine Research, National Health Research Institutes, Miaoli, Taiwan, "The Department \\ of Laboratory Medicine, National Taiwan University Hospital, Taipei, Taiwan
}

To read the full text of this article, go to http://www.fasebj.org/cgi/doi/10.1096/fj.05-4288fje; doi: 10.1096/fj.05-4288fje

\section{SPECIFIC AIMS}

One of the major problems inherent to the study in the use of stem cells in tissue regeneration is the difficulty in tracking the movement of implanted cells. There has been significant effort to develop new nanoparticle vectors that could be efficiently internalized into cells. However, current cellular labeling apparatus are barely satisfactory. We sought to develop a highly efficient cell labeling nanoparticle vector for stem cell tracking.

\section{PRINCIPAL FINDINGS}

1. Fluorescein isothiocyanate-conjugated mesoporous silica nanoparticles (FITC-MSNs) exhibit highly efficient cell labeling activity

For this study, we synthesized mesoporous silica nanoparticles and labeled them with fluorescein isothiocyanate for detection. As revealed by transmission electron microscope (TEM) examination, FITC-MSNs had welldefined hexagonal edges and highly ordered porous and were uniform in size $(\sim 110 \mathrm{~nm})$. We tested whether FITC-MSNs would be internalized into cells and subsequently determined the mechanism and the effect of internalized FITC-MSNs on human mesenchymal stem cells (hMSCs) as well as on 3T3-L1 cells, which is an established cell line and would differentiate into adipocyte under appropriate conditions, for comparison. As demonstrated by flow cytometric analysis, FITCMSNs were internalized into both cells in a time- and concentration-dependent manner. The FITC-MSN uptake began efficiently as early as $10 \mathrm{~min}$ after incubation and was relatively rapid within the first $1-2 \mathrm{~h}$ of incubation, and a saturation uptake was achieved at $2 \mathrm{~h}$ and 2-4 h of incubation in hMSCs and 3T3-L1 cells, respectively. Moreover, at higher doses of FITC-MSN treatment, the histogram of fluorescence intensity exceeded the intensity scale indicating a very efficient uptake of FITC-MSNs. Confocal analysis revealed that FITC-MSNs were internalized into cells indeed (Fig. 1A). TEM images showed that FITC-MSNs retained the architecture integrities including highly ordered hexagonal pores, well-defined hexagonal edges, and particle size after their internalization into cells (Fig. $1 B$ ).

\section{The internalized FITC-MSNs do not affect cell viability, growth, and differentiation}

We next explored the effects of FITC-MSNs on cellular activities, including cytotoxicity, cell proliferation, and cellular differentiation activities. Neither evidence of cytotoxicity was observed at $1 \mathrm{~h}$ after treatment with FITC-MSNs nor was the cell proliferation affected after FITC-MSN-treated cells were incubated with growth medium for 1-3 days. After treatment with FITC-MSNs, identical profiles of immunophenotype detected by flow cytometry were noticed in FITC-MSN-labeled or control hMSCs. FITC-MSN-treated hMSCs retained their differentiation activities into adipocytes, chondrocytes, and osteocytes like untreated control cells. FITCMSN-treated 3T3-L1 cells exerted the identical adipogenic differentiation as control cells.

\footnotetext{
${ }^{1}$ Correspondence: Department of Laboratory Medicine, National Taiwan University Hospital, No. 7, Chung-Shan South Road, Taipei 100, Taiwan. E-mail: ycchenmd@ha. mc.ntu.edu.tw
} 
A
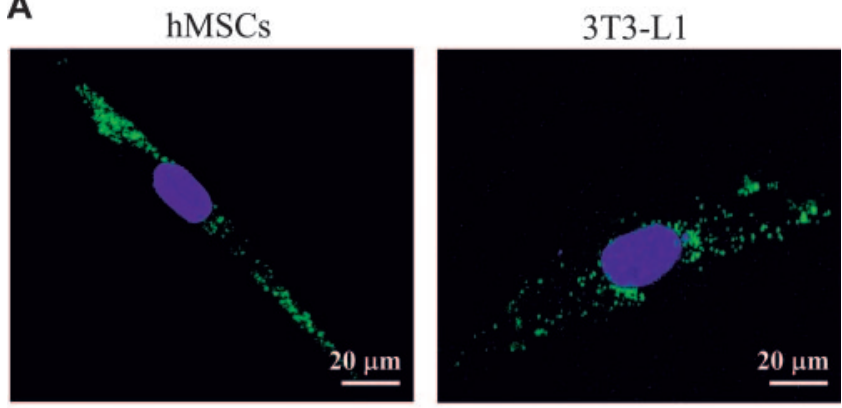

B

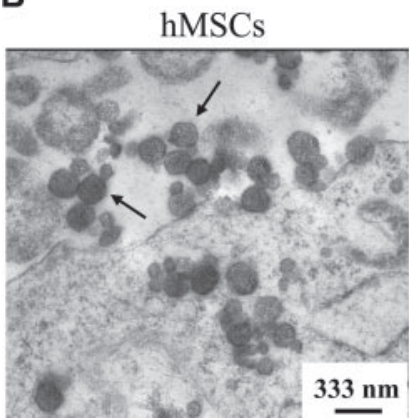

3T3-L1

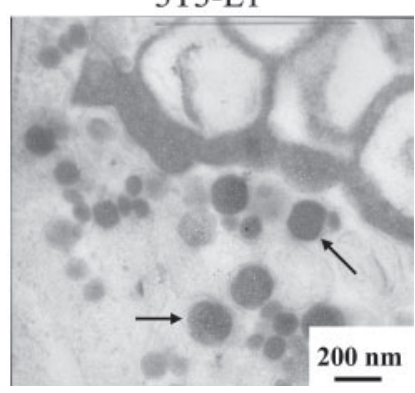

Figure 1. Microscopic observation of the FITC-MSN internalization. hMSCs (left panels) and 3T3-L1 cells (right panels) were treated with FITC-MSNs for $1 \mathrm{~h}$, then processed for confocal microscopic examination $(A)$ or TEM study $(B)$. $A$ ) Cell nucleus was stained with 4',6-diamidino-2-phenylindole (blue color). FITC-MSNs appeared in green color. B) FITC-MSNs (arrows) exhibited hexagonal figures after internalization into cells.

\section{Clathrin-mediated endocytosis plays an important} role in the FITC-MSN uptake and is involved in more uptake and longer stay of FITC-MSNs in hMSCs than in $3 \mathrm{~T} 3-\mathrm{L} 1$ cells

Inhibition of clathrin-coated pit endocytosis by sucroseinduced hypertonicity and phenylarsine oxide, a clathrin inhibitor, decreased FITC-MSN uptake, whereas inhibition of caveola-coated pit endocytosis by filipin did not affect FITC-MSN uptake. We examined the correlation between clathrin protein expression and the uptake and stay duration of FITC-MSNs in 3T3-L1 cells and hMSCs. 3T3-L1 cells displayed less FITC-MSN uptake and a shorter period of FITC-MSN stay as well as reduced clathrin protein expression than hMSCs.

\section{The internalized FITC-MSNs can escape from} endocytic vesicles (endo-lysosomal vesicles) and resist the lysosomal degradation

Since in the endocytic pathway ingested molecules are delivered to early endosomes, then (via late endosomes) to lysosomes, we studied the colocalization of LysoTracker Red, a marker for late endosomes and lysosomes with red fluorescence, with FITC-MSNs (green fluorescence). A partial colocalization (orange to yellow fluorescence) in the cytoplasmic compartment was exhibited (Fig. 2A). However, FITC-MSNs

appeared to accumulate in compartments separated from the LysoTrack-labeled compartments. As shown in Fig. 2B, we found that some internalized FITC-MSNs were colocalized with nonyl acridine orange (NAO, a mitochondria-selective probe) in the mitochondria, as evident from the appearance of yellow fluorescence. As demonstrated in Fig. $1 B$, the maintenance of architecture integrities of FITC-MSNs after their internalization into cells indicated that FITC-MSNs were able to resist the lysosomal degradation.

\section{CONCLUSIONS AND SIGNIFICANCE}

Since certain nanoparticle vectors commonly used to label cells have significant limitations, low cellular internalizing efficiency especially, there is a strong interest in the development of highly efficient cellular labeling vectors. To our knowledge current vectors for cell labeling or tracking are composed of biodegradable nanoparticles. In the present study, we attempted to label cells with mesoporous silica nanoparticles
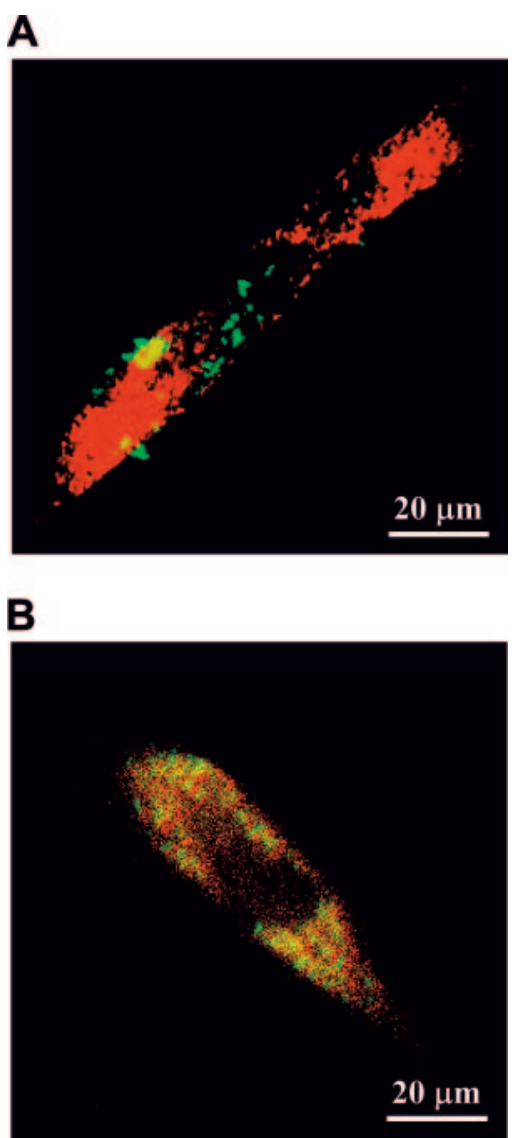

Figure 2. Colocalization of green fluorescent FITC-MSNs with late endosomes/lysosomes and mitochondria. hMSCs were treated with FITC-MSNs for $30 \mathrm{~min}$, then incubated with LysoTracker Red $(A)$ or nonyl acridine orange (NAO) $(B)$ for another $30 \mathrm{~min}$. The cellular distribution of FITC-MSNs and LysoTracker Red- or NAO-labeled organelles (late endosomes/lysosomes and mitochondria, respectively) was analyzed by a Zeiss Axiovert 100M confocal unit. 


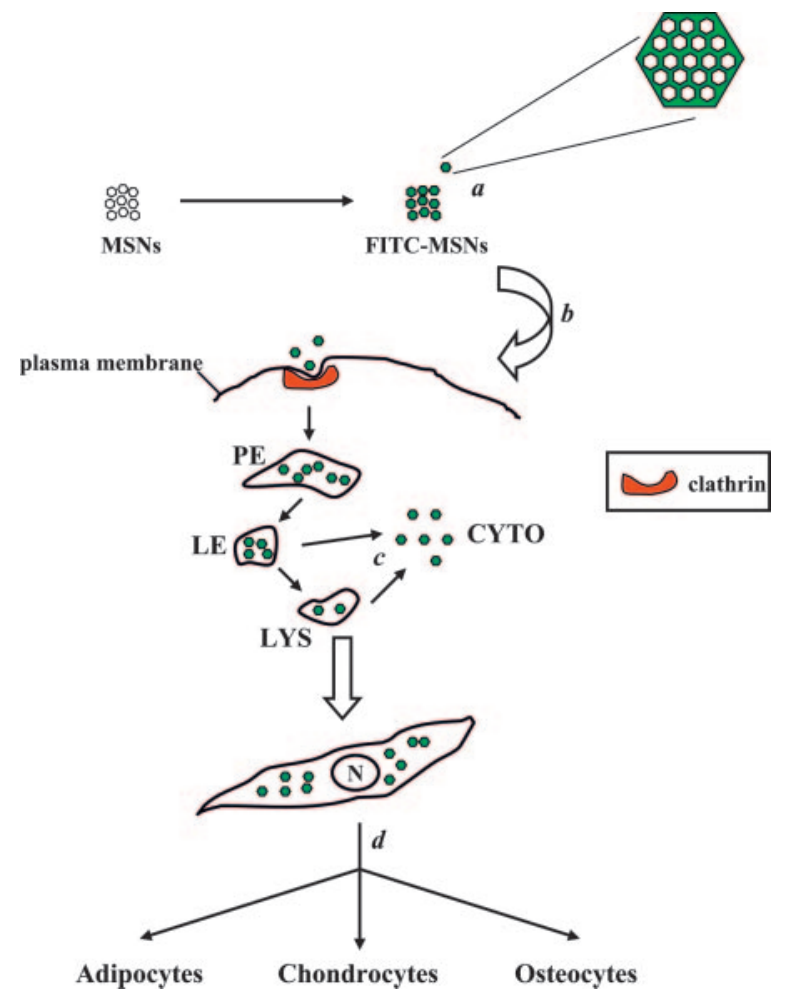

Figure 3. Schematic representation of the intracellular internalization and endolysosomal escape of FITC-MSNs. a) FITCMSNs have well-defined hexagonal edges and highly ordered porous. $b$ ) Clathrin-mediated endocytosis plays an important role in the FITC-MSN uptake and is involved in more uptake and longer stay of FITC-MSNs in hMSCs than in 3T3-L1 cells. c) After their internalization, FITC-MSNs (depicted as closed green circles) are transported to late endosomes (LE) and lysosomes (LYS) via early or primary endosomes (PE), then escape from endo-lysosomal vesicles into the cytoplasm (CYTO) and resist the lysosomal degradation. $d$ ) The internalized FITC-MSNs have no inhibitory effect on cell viability, growth, and differentiation. $\mathrm{N}$, nucleus.

(MSNs), which are considerably nonbiodegradable, and evaluated the possibility of MSNs for being a cell tracker, especially for stem cell tracking.

In the present study, we successfully labeled hMSCs and 3T3-L1 cells with FITC-MSNs. Without any modification, FITC-MSNs could be internalized into cells efficiently as evidenced by a swift occurrence of uptake detected by flow cytometry in a concentration- and time-dependent manner. The FITC-MSN internalization occurred within the ten-minute treatment and substantially accumulated during the first two-hour incubation. Microscopic examinations demonstrated that the intracellular internalization determined by flow cytometry was not due to the adherence to cell surface. As compared with 3T3-L1 cells, hMSCs internalized FITC-MSNs more efficiently and reserved the intracellular FITC-MSNs longer, and this finding implies that FITC-MSNs could be an appropriate candidate for the use of stem cell tracking. We also demonstrated that FITC-MSN internalization did not affect cell viability, cell proliferation, and the immunophenotypic profiles of surface markers in hMSCs. The labeled hMSCs also retained their multipotency to differentiate into fat (adipocytes), cartilage (chondrocytes), and bone (osteocytes) after cellular internalization of FITCMSNs, and the labeled 3T3-L1 cells exerted equal adipogenic differentiation ability to the untreated cells. These facts indicate that FITC-MSNs are quite biocompatible.

The results also indicate that clathrin-mediated endocytosis plays an important role in the FITC-MSN uptake and contributes to the more uptake and longer stay of FITC-MSNs in hMSCs than in 3T3-L1 cells. After intracellular uptake by endocytosis, FITC-MSNs escape out of the endolysosomes from degradation. Another possibility of contribution to the resistance to lysosomal degradation is due to the nonbiodegradability of silica. However, the nonbiodegradability is not harmful to the bioactivities of cultured cells in vitro as well as in animal mode (data not shown).

Our data demonstrate that: 1) the mesoporous silica nanoparticles can be internalized efficiently into hMSCs and 3T3-L1 cells by clathrin-dependent endocytosis; 2) The mesoporous silica nanoparticles are nontoxic without affecting cell viability, growth, and differentiation; 3) Human mesenchymal stem cells can internalize more mesoporous silica nanoparticles and keep them inside longer; and 4) The mesoporous silica nanoparticles can escape from endo-lysosomal vesicles and resist lysosomal degradation. Although further investigations are warranted to evaluate the potential use of mesoporous silica nanoparticles, these results at least suggest the potential application of these nanoparticles in stem cell tracking. 